\title{
Wavelet Transform in Remote Sensing Image Compression of the Key Technical Analysis
}

\author{
Liu Yanyan \\ Institute of Electronic and Information Engineering, Changchun University of Science and Technology \\ Room 318, BLD 1, No.7089, Weixing Road, Changchun 130022, Jilin, China \\ Tel: 86-431-8558-2269Ｅ-mail: liuyy306@163.com \\ Li Guoning \\ Changchun Institute of Optics, Fine Mechanics and Physics, Chinese Academy of Sciences \\ No.3888, Nanhuda Road, Changchun 130033, Jilin, China \\ Tel: 86-431-8670-8862 E-mail: lgning@netease.com
}

Received: December 22, 2011 Accepted: January 19, $2012 \quad$ Published: April 1, 2012

doi:10.5539/mas.v6n4p44 URL: http://dx.doi.org/10.5539/mas.v6n4p44

\begin{abstract}
To solve the algorithm in the image compression of remote sensing than complex, efficiency lower etc. problem, this paper has put forward a kind of fast, efficient image compressed algorithm on the foundation of little wave coding. Firstly, have gone on for the aspects such as the image compressed coding method, kind and performance appraisement of remote sensing have been further elaborated. Then the coding based on wavelet transform has been described in detail. Finally crucial technology for the image compressed algorithm of remote sensing has carried out thorough research. Experiment result shows: The PSNR value of the image compressed algorithm of remote sensing that this paper adopts has got great advance than traditional algorithm.
\end{abstract}

Keywords: Remote sensing image, Compression coding, Wavelet transform, JPEG2000

\section{Introduction}

Now, the algorithm that applies to the image compressed coding of remote sensing includes mainly: Foresee coding and dispersed cosine coding (DCT) as well as dispersed wavelet transform coding (DWT), their neutral can superior coding technology will apply still in the following image compressed coding system of remote sensing (Pen S. Y., 2005; Wu W. B., 2010).

Along with the increase of the image data of remote sensing, the requirement for the image compressed algorithm of remote sensing is also high (Sui Y. P., 2008). How to reduce the complex degree of image compressed algorithm, how to guarantee image compressibility, which is the present problem that need to solve. Research applies to the compressed algorithm of the image of remote sensing to have important theoretical meaning and project application value (Qi C. D., Chen L. \& Zeng T., 2009).

According to the characteristic of the image compression of remote sensing and requirement, this paper have put forward a kind of fast and efficiently compressed algorithm that applies to the image of remote sensing on foundation of wavelet transform.

\section{Wavelet Transform Remote Sensing Image Compression Algorithm}

\subsection{The Development of Wavelet Transformation}

The concept of wavelet transform, by the French scientists put forward in the 1970s. In 1988s, Mallat in tectonic orthogonal wavelet base, puts forward the much solution concept, and explained the image wavelet much solution characteristics. He put forward the constructive method of orthogonal wavelet and fast algorithm, which Mallat algorithm, So that the discrete wavelet analysis into reality. Through a fixed function after translation and expansion and get the wavelet method, called the first generation wavelet transformation and Fourier transform is to build the first generation of wavelet transform the most essential tool (Wang M. F., et al., 2009). 


\subsection{Based on Wavelet Transformation of the Coding Algorithm}

Assume that the original image by a series of pixel set PI, $\mathrm{j}$ definition, among them (I, j) said in the images of the pixel on the space coordinates, symbol $\mathrm{p}$ says the original image array. Symbols $\mathrm{c}$ said $\mathrm{p}$ after orthogonal transformation of after $2 \mathrm{~d}$ coefficients array, ci, $\mathrm{j}$ said after the orthogonal transformation coordinates $(\mathrm{I}, \mathrm{j})$, the transform coefficients.

In the process of transmission, first passed the most important image information, and then transfer times important factor, that is, according to the order of importance image encoder information transmission (Deng J. X., 2006; Gerek O. N., Cetin A. E., 2006). When decoder to receive any given a bit rate of the image compression data, can make the distortion degree of the reconstruction of the image is the smallest. Evaluation of compressed image restoration using mean square error (MSE) to say, such as type (1).

$$
D(p-\hat{p})=\frac{\|p-\hat{p}\|^{2}}{N_{1} N_{2}}=\frac{1}{N_{1} N_{2}} \sum_{i=0}^{N_{1}-1} \sum_{j=0}^{N_{2}-1}\left(p_{i, j}-\hat{p}_{i, j}\right)^{2}
$$

Among them, the N1 said the image line number, N2 said column number of the image. For unit for orthogonal transformation, transform the before and after Euclid norm constant, so have type (2) is founded.

$$
D(p-\hat{p})=D(c-\hat{c})=\frac{1}{N_{1} N_{2}} \sum_{i=0}^{N_{1}-1} \sum_{j=0}^{N_{2}-1}\left(c_{i, j}-\hat{c}_{i, j}\right)^{2}
$$

From the formula (2) can see, when transform coefficients ci, $\mathrm{j}$ accurate sent to the after image decoder, reconstruction images of the mean square error decreases. It can be concluded that the image compression in the process of streaming output, when the bit rate or distortion degree to the expected requirements, compression terminated. When using the importance to send information based on to the gradual transfer method, in any code rate three wavebands of image information distortion degree can minimum.

\section{The Key Technical Analysis of Image Coding}

Based on the new application needs to make the proposal of the standard JPEG2000 is at first in March 1997 was proposed.

\subsection{JPEG2000 Coding Structure}

JPEG2000 coding structure as shown in Figure 1 of shows, mainly including pretreatment, discrete wavelet transform (DWT), and quantization (the compression don't need) and optimizing the truncation embedded piece of code (EBCOT), etc. In order to realize the compression and loss compression two kind of coding, it used respectively integral wavelet and floating point of wavelet transform and corresponding. And JPEG standard of use discrete cosine coding is different, use is JPEG2000 wavelet transform code, which is also has excellent performance is one of the main reasons (Zheng C. L., et al., 2010; Liu Y. Z., et al., 2009).

\subsubsection{Pretreatment}

The pretreatment of object is the original image, it mainly includes the images of rectangular (tile) segmentation, DC level (DC) translation and component transform three parts.

(1) Image segmentation rectangular piece

Image compression encoder will allow an image into smaller image piece, each image of compression encoder operational independence unit. Generally speaking, the image of rectangular divided, in addition to the edges area outside, every image of the size of the piece is same.

\section{(2) DC translation}

If the image data samples value is negative, quantitative digits is $P$, so they are the center of the dynamic range is not zero, they require DC pan, just put all samples values are the offset value minus the can. This input image data sampling of the values of the dynamic range from into. When the input image data sampling value transform came to zero as the center of the range, so in the code you can to ensure that the data will not overflow, etc. When the input with signs samples value, they don't need to DC translation.

\section{(3) Component transformation}

Component transform is in DC after translation. It applies to all of the component, main effect is to reduce the correlation of the seismic data, so as to improve the efficiency of the code (C. Christopoulos, A. Skodras, \& T. Ebrahimi., 2000).

In JPEG2000 standards, there are two kinds of forms of component transformation: irreversible component 
transform (ICT) and reversible component transform (RCT). But to gray image, because there is only one component pixel sampling, and therefore do not need weight change. Reversible component of transformation is defined as transformation formula (3) and (4) is it formula of inverse transform:

$$
\begin{gathered}
{\left[\begin{array}{c}
Y \\
C_{b} \\
C_{r}
\end{array}\right]=\left[\begin{array}{ccc}
0.299 & 0.587 & 0.114 \\
-0.16875 & -0.33126 & 0.5 \\
0.5 & -0.41869 & -0.08131
\end{array}\right] \cdot\left[\begin{array}{l}
R \\
G \\
B
\end{array}\right]} \\
{\left[\begin{array}{l}
R \\
G \\
B
\end{array}\right]=\left[\begin{array}{ccc}
1.0 & 0 & 1.402 \\
1.0 & -0.34413 & -0.71414 \\
1.0 & 1.772 & 0
\end{array}\right] \cdot\left[\begin{array}{c}
Y \\
C_{b} \\
C_{r}
\end{array}\right]}
\end{gathered}
$$

Irreversible component of transformation is defined as transformation formula (5) and formula (6) is it formula of inverse transform:

$$
\begin{aligned}
& {\left[\begin{array}{c}
Y r \\
U r \\
V r
\end{array}\right]=\left[\begin{array}{c}
\left\lfloor\frac{R+2 G+B}{4}\right\rfloor \\
R-G \\
B-G
\end{array}\right]} \\
& {\left[\begin{array}{c}
G \\
R \\
B
\end{array}\right]=\left[\begin{array}{c}
Y r-\left\lfloor\frac{U_{r}+V_{r}}{4}\right\rfloor \\
U r+G \\
V_{r}+G
\end{array}\right]}
\end{aligned}
$$

\subsubsection{Discrete Wavelet Transformation}

After weight after the transformation, the pictures in the image data for a unit of discrete wavelet transform, the wavelet transform image data in different resolution on the decomposition. In the resolution of the different level, have a lot of sub band, these sub band describes the original image in a horizontal and vertical direction of frequency characteristics.

\subsubsection{Quantitative}

For the compression, wavelet coefficient do not need to undertake the quantization process, quantification is only for lossy compression. The scalar quantification is JPEG2000, quantitative aim is to the wavelet coefficients range from interval mapped to a range between more areas, reduce the precision of the coefficient, and better compression. For the son take b, its coefficient quantification of formula (7) shown.

$$
q_{b}(u, v)=\operatorname{sign}\left(a_{b}(u, v)\right)\left\lfloor\frac{\left|a_{b}(u, v)\right|}{\Delta_{b}}\right\rfloor
$$

Type in the symbols take coefficient said, is the son of the step length with quantitative.

\subsubsection{EBCOT Encoder}

By the first layer EBCOT encoder entropy coding (Tier 1) and the second code (Tier 2) composition. Tier 1 for each piece of complete (code-block) coding, it includes a plane of coding (fractional bit-plane coding) and MQ encoder two parts. Tier 2 major final data streaming organization, including streaming stratified and packaging, operation.

\subsection{The Experimental Results}

In order to obtain in different bit rate of compressed image, should to the final code flow truncation, each coding information is the end of truncated points. From the Figure 2 can be found in the same code rate, under the condition of the solid line of the corresponding coding performance was significantly higher than the dotted line. Therefore, in order to make the same bit rate environment reconstruction images of distortion as far as possible the small, should will rate distortion slope big coding information adjust to the front. 
For a range of truncated point, take a certain value, makes getting minimum value, that is without any increase in overall bit rate, distortion has the smallest.

$$
J(\lambda)=\sum_{i}\left(R_{i}^{n_{i}^{\lambda}}+\lambda D_{i}^{n_{i}^{\lambda}}\right)
$$

\section{Conclusion}

In the object code rate is low, have been coding to a large number of streaming will be discarded, eventually did not included in the compression streaming, which causes to forsake streaming coding waste. Therefore, the same picture in all kinds of code rate under code JPEG2000, the complexity of the code and the processing time almost are not significant.

For CCD image and respectively remote sensing image, in all kinds of code rate under the detailed test, and give the image PSNR experimental results. The experiment results were shown in Figure 3 and Figure 4. As contrast is given, and the software realization JPEG2000 test results PSNR. Compression interface card processing unit can be compressed all the way the image data rate of $204.68 \mathrm{Mbps}$. The actual test results show that the compression system meet the prescribed technical index.

\section{References}

Christopoulos, C., Skodras, A., \& Ebrahimi, T. (2000). The JPEG2000 still image coding system an overview. IEEE Transactions on Consumer Electronics, 46(4), 1103-1127. http://dx.doi.org/10.1109/30.920468

Deng, J. X. (2006). A remote--sensing image coding algorithm based on significant coefficients lifting. Optics and Precision Engineering, 14(5), 910-916.

Gerek, O. N., \& Cetin, A. E. (2006). 2-D orientation - adaptive prediction filter in lifting structures for image coding. IEEE Transactions on Image Processing, 15(1), 106-1111. http://dx.doi.org/10.1109/TIP.2005.859369

Liu, Y. Z., et al. (2009). The Design of JPEG2000 Still Image Compression System Based on ADV212. Electron Devices, 32(3), 504-508.

Pen, S. Y., Philippe, A., Aaron, K., et al. (2005). The New CCSDS Image Compression Recommendation. IEEE Aerospace Conference, Berlin, 5-10, 4138-4145.

Qi, C. D., Chen, L., \& Zeng, T. (2009). Multi-channel Image Data of CCD Real-time Record System. Opto-Electronic Engineering, 36(12), 147-150.

Sui, Y. P., et al. (2008). A compression algorithm of remote sensing image based on ROI for ocean surveillance satellite. Optics and Precision Engineering, 16(7), 1323-1329.

Wang, M. F., et al. (2009). An Image Compression Algorithm for Remote Sensing Image Based on Wavelet Transform. Opto-Electronic Engineering, 36(11), 85-90.

Wu, W. B. et al. (2010). Research on Application of CCSDS in Remote Sensing Image Compression. SPACECRAFT RECOVERY \&REMOTE SENSING, 31(2), 46-50.

Zheng, C. L., et al. (2010). Design for Unmanned Aerial Vehicle Image sequence Compressing System Based on ADV202. Computer \& Digital Engineering, 38(11), 136-141.

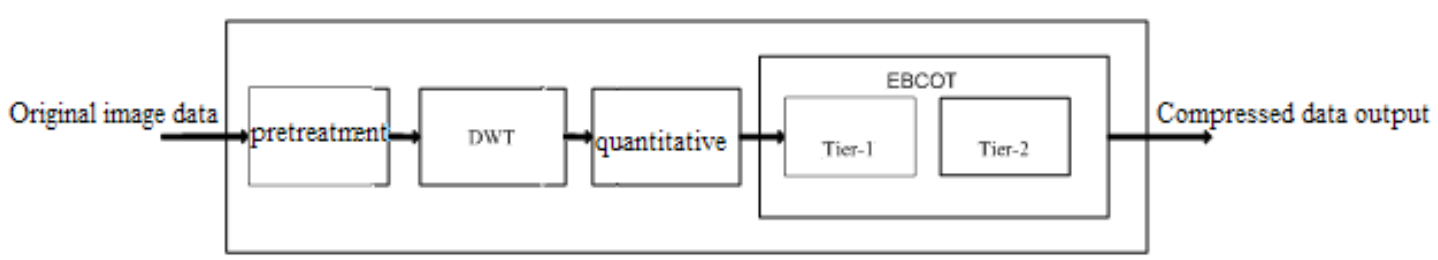

Figure 1. JPEG2000 encoder structure (join rate distortion) 


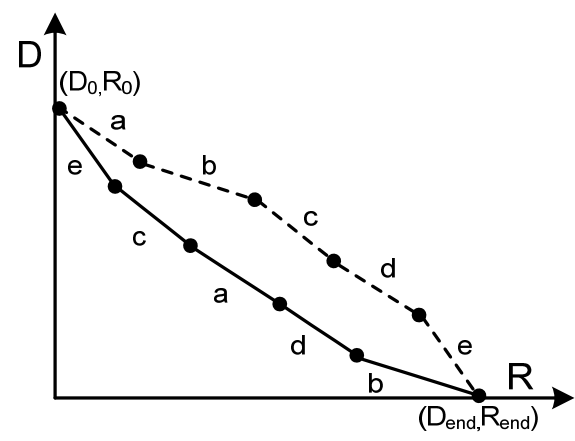

Figure 2. Rate distortion optimization schemes

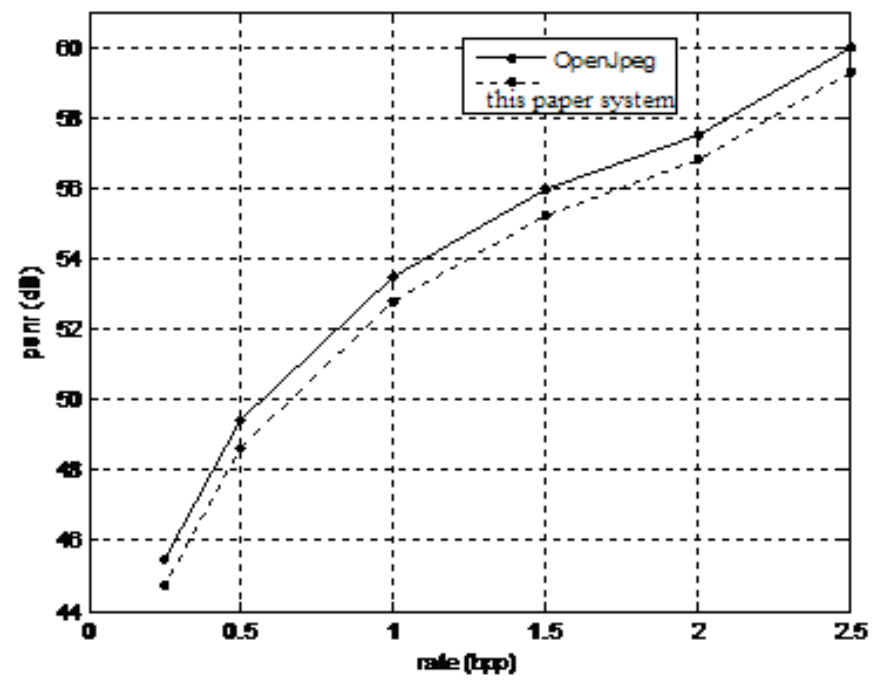

Figure 3. CCD image contrast test of PSNR average curve

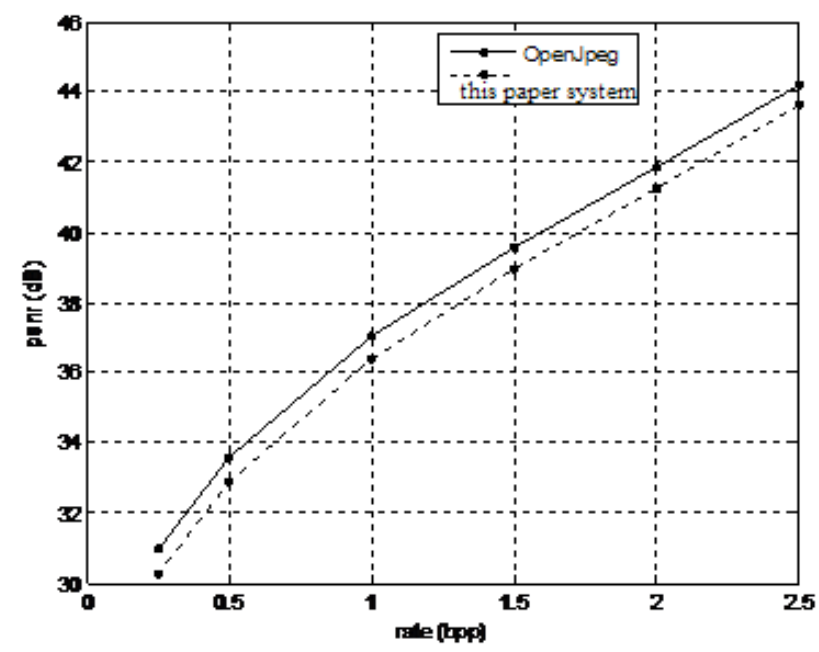

Figure 4. Remote sensing image contrast curve of PSNR average 